\title{
Impact of Eccentricity on Nonlinear Oscillations of a Point-Like Charge in the Electric Field of a Curvature-Dependent Elliptic Charged Ellipse
}

\author{
Haiduke Sarafian \\ The Pennsylvania State University, University College, York, USA \\ Email: has2@psu.edu
}

How to cite this paper: Sarafian, H. (2020) Impact of Eccentricity on Nonlinear Oscillations of a Point-Like Charge in the Electric Field of a Curvature-Dependent Elliptic Charged Ellipse. American Journal of Computational Mathematics, 10, 603-611. https://doi.org/10.4236/ajcm.2020.104035

Received: November 20, 2020

Accepted: December 18, 2020

Published: December 21, 2020

Copyright $\odot 2020$ by author(s) and Scientific Research Publishing Inc. This work is licensed under the Creative Commons Attribution International License (CC BY 4.0).

http://creativecommons.org/licenses/by/4.0/ (c) (i) Open Access

\begin{abstract}
Calculation of the interactive force between two horizontally stacked circular uniformly charged rings placed along the common vertical axis conducive to nonlinear oscillations under gravity has been addressed [1]. Although challenging, nonetheless the scope of the study limited to uniform charge distributions of the rings. Here we extend the analysis considering a charged ellipse with a nonuniform, curvature-dependent elliptic charge distribution exerting a force on a point-like charge placed on the vertical symmetry axis. Nonuniform charge distribution and its impact on various practical scenarios are not a common theme addressed in literature. Applying Computer Algebra System (CAS) particularly Mathematica [2], we analyze the issue on hand augmenting the traditional scope of interest. We overcome the CPU expensive symbolic computation following our newly developed numeric/symbolic method [1]. For comprehensive understanding, we simulate the nonlinear oscillations.
\end{abstract}

\section{Keywords}

Nonlinear Oscillations, Elliptic Curvature-Dependent Charge Distribution, Computer Algebra System, Mathematica

\section{Introduction, Motivations and Goals}

Calculating the electric field of a uniform charge distribution on common geometric objects such as circle, rectangle, square, rumbas, etc., among others along their vertical symmetry axis is trivial [3]. However, for instance calculating the field for objects with less symmetries e.g. an ellipse vs. a circle is challenging; in general, "less the symmetry challenging the issue." In our particular instance, the nonuniform curvature breaks the symmetry. Having assumed this issue can be 
addressed one might wonder about its practical applications. To this end motivated with the outlined procedure [1], here we consider a scenario where a curvature-dependent elliptic charge distribution interacts with a point-like charge resulting nonlinear oscillations. Figure 1 visually clarifies the issue on hand.

The shown scenario in Figure 1 visually describes the issue on hand. A curvature-dependent elliptic charge distribution makes an electric field. We focus only on the field along the vertical symmetry axis where a point-like massive particle of the same color as the ellipse is placed. Naturally, the massive, charged particle gets pulled under the gravity encountering a repulsive electric force via the elliptic charge. Theoretically, it is viable to visualize a situation that by adjusting the relevant physical parameters such as the charges and the mass of the particle on one hand and the geometrical parameters such as the size of the ellipse as well as the initial position of the point charge and its initial velocity make the aforementioned forces compatible resulting oscillations. And, therefore, it is the goal of this investigation to quantifying the characteristics of the oscillations conducive to stable vibrations, identifying amplitude, period and related relevant characters. It is also desired to compare the oscillations for different ellipses characterized by their respective eccentricities, henceforth, comparing the oscillations of the elliptic charge distributions to a circular, uniform distribution. The latter would reveal the impact of the nonuniform vs. the uniform distribution. This article is composed of three sections. In addition to Sect. 1, Introduction, Motions and Goals in Section 2 we lay down the Mathematical formal development of the issues on hand. This section also embodies computer codes. As mentioned, we have applied a Computer Algebra System (CAS), particularly Mathematica to carry out the needed symbolic and numeric computations. And hence, codes are crafted in Mathematica language. Interested readers may apply the codes producing our result.

To begin with, the needed parameters are stored in the listed values1, units are all in SI, the values are feasibly practical.

$$
\text { values } 1=\left\{k \rightarrow 9.10^{9}, \mathrm{q} 1 \rightarrow 1.10^{-6}, \mathrm{q} 2 \rightarrow 2.10^{-6}, \mathrm{a} \rightarrow 2 ., \mathrm{b} \rightarrow 0.5, \mathrm{~m} \rightarrow 0.110^{-3}, \mathrm{~g} \rightarrow 9.8\right\} ;
$$

The listing includes the electrostatic coupling constant, $k=\frac{1}{4 \pi \epsilon_{0}}=9 \times 10^{9}$ with $\in_{0}$ being the permittivity of vacuum, $q_{1}$ is the charge on the ellipse. The loose point-like particle has a mass $m$ and charge $q_{2}$. The $a$ and $b$ are the semi major and minor lengths of the ellipse, respectively with implicit eccentricity $e=\sqrt{1-\left(\frac{b}{a}\right)^{2}}, e=0.96$ [4]. This large $e$ indicates the ellipse is stretched along the major axis, $\mathrm{x}$-axis, as opposed to the minor axis, $\mathrm{y}$-axis. Numeric values of the axes have chosen such that $a \gg b$ with an attempt magnifying the impact of the uneven charge distribution. In practice by enlarging the $b$ the ellipse reshapes approaching a circle causing the uneven distribution runs to even, uniform distribution with well-known consequences such as e.g. electric field [3]. The values of the charges and the size of the ellipse although chosen arbitrarily 


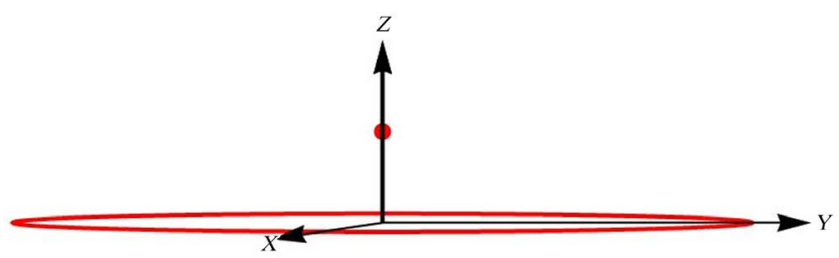

Figure 1. An ellipse with curvature-dependent elliptic charge distribution is clamped on a horizontal support with a point-like massive charge placed on its symmetry axis through the center. The ellipse and the charge are positively charged.

is practical. However, the mass of the particle ought to be determined compatibly to fulfilling the objective of the search: oscillations. An ill-posed mass would cause catastrophe; the particle falls through the ellipse with no chance of return more on this in follow-up paragraphs.

It is beneficiary quantifying the latter paragraph. The code below generates two ellipses and their associated curvatures.

plotellipse1=ParametricPlot $[\{\mathrm{a} \operatorname{Cos}[\theta], \mathrm{b} \operatorname{Sin}[\theta]\} /$.values $1,\{\theta, 0,2 \pi\}$, AxesLabel ->\{"x","y"\},GridLines->Automatic,PlotStyle->Black];

plotellipse2=ParametricPlot $[\{\mathrm{a} \operatorname{Cos}[\theta], \mathrm{b} \operatorname{Sin}[\theta]\} /$.values $2,\{\theta, 0,2 \pi\}$, AxesLabel ->\{"x","y"\},GridLines->Automatic,PlotStyle->\{Dashing[\{0.01\}],Black\}];

plotEllipses21=Show [\{plotellipse2,plotellipse1\}];

plotcurature $1=\mathrm{Plot}\left[(\mathrm{a} b) /\left((\mathrm{a} \operatorname{Sin}[\theta])^{\wedge} 2+(\mathrm{b} \operatorname{Cos}[\theta])^{\wedge} 2\right)^{3 / 2} 1 / 2(\mathrm{a}+\mathrm{b}) /\right.$. values 1 , $\{\theta, 0,2 \pi\}$,PlotRange- $>$ All,GridLines- $>\{\{0,1 / 2 \pi, \pi, 3 / 2 \pi, 2 \pi\}$,Automatic $\}$, AxesOrigin- $>\{0,0\}$, Ticks- $>\{\{0,1 / 2 \pi, \pi, 3 / 2 \pi, 2 \pi\}$, Automatic $\}$, AxesLabel ->\{"$\theta(\mathrm{rad}) "$, "Curvature" $\},$ PlotStyle->Black];

plotcurature $2=\operatorname{Plot}\left[(\mathrm{a} b) /\left((\mathrm{a} \operatorname{Sin}[\theta])^{\wedge} 2+(\mathrm{b} \operatorname{Cos}[\theta])^{\wedge} 2\right)^{3 / 2} 1 / 2(a+b) /\right.$. values2, $\{\theta, 0,2 \pi\}$, PlotRange- $>$ All,GridLines- $>\{\{0,1 / 2 \pi, \pi, 3 / 2 \pi, 2 \pi\}$, Automatic $\}$, AxesOrigin- $>\{0,0\}$, Ticks- $>\{\{0,1 / 2 \pi, \pi, 3 / 2 \pi, 2 \pi\}$, Automatic $\}$,AxesLabel ->\{"$\theta(\mathrm{rad}) "$, "Curvature" $\},$ PlotStyle->\{Dashing[\{0.01\}],Black $\}]$; plotCurvature21=Show [ plotcurature2, plotcurature1\}];

As shown in Figure 2 there is a one-to-one correspondence between the left and the right panels. As such, the solid elliptic curve on the left panel with sharp edges along the $\mathrm{x}$-axis corresponds to high curvature values on the right panel at three polar angles, $0, \pi$, and $2 \pi$ with $\pi$ radian cyclic repetition angle. Conversely, the soft curved segment of the ellipse at the edges of the minor axis corresponds to the low curvature on the right panel at two polar angles $\pi / 2$, and $3 / 2 \pi$ with a $\pi$ radian cyclic repetition polar angle. The same general feature holds for the dashed ellipse; however, the curvature values are less than the previous case due the smaller eccentricity.

As the second step we focus characterizing the linear-charge density of the ellipse. Accordingly, we modify the basic definition of density incorporating the elliptic curvature,

$$
\lambda(\theta)=\frac{q_{1}}{\pi[3(a+b)-\sqrt{(3 a+b)(a+3 b)}]} \frac{a b}{\left[(a \sin [\theta])^{2}+(b \cos [\theta])^{2}\right]^{\frac{3}{2}}} \frac{1}{2}(a+b)(1)
$$



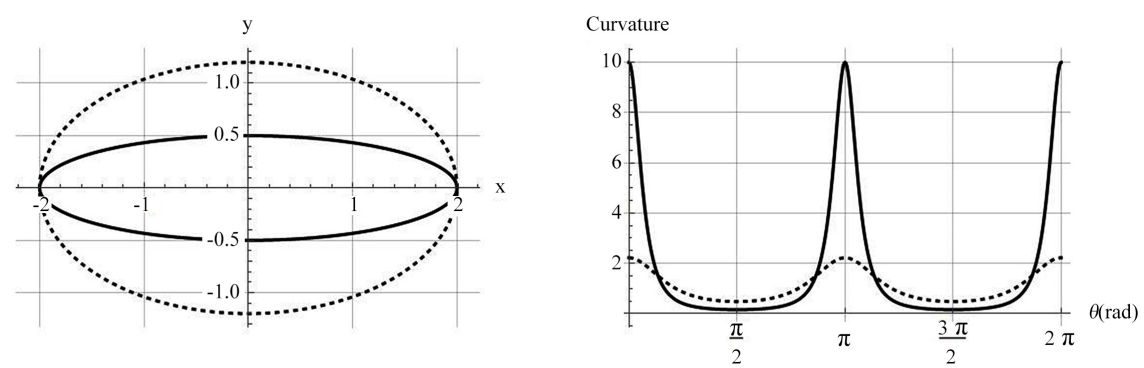

Figure 2. Display of two different ellipses with eccentricities 0.96 (solid) and 0.80 (dashed), respectively. The right panel is their corresponding curvatures.

The denominator of the first term in (1) is the approximate length of the ellipse's circumference [5]. The second term is the modeled elliptic curvature [4], with $\theta$ being the polar angle parametrizing the ellipse. The third term is an average value of the size of the ellipse, this is included making the dimension of $[\lambda]=$ charge/length. Since $\lambda$ ought to be normalized to the charge on the ellipse, $q_{1}$ i.e. $\int \lambda(\theta) \mathrm{d} \ell=q_{1}$ with $\mathrm{d} \ell=\sqrt{(a \sin [\theta])^{2}+(b \cos [\theta])^{2}} \mathrm{~d} \theta$ we introduce a normalization factor enforcing this characteristic. This factor for listed values 1 is,

normalizationFactor $1=\operatorname{NIntegrate}[1 /(\pi(3(a+b)-\sqrt{ }((3 a+b)(a+3 b)))) 1 / 2(a+b)$ $\left.(\mathrm{a} \mathrm{b}) /\left((\mathrm{a} \operatorname{Sin}[\theta])^{\wedge} 2+(\mathrm{b} \operatorname{Cos}[\theta])^{\wedge} 2\right)^{3 / 2} \sqrt{ }\left((\mathrm{a} \operatorname{Sin}[\theta])^{\wedge} 2+(\mathrm{b} \operatorname{Cos}[\theta])^{\wedge} 2\right)\right) /$. values 1 , $\{\theta, 0,2 \pi\}]$

So that the normalized $\lambda(\theta)$ is,

$\lambda 1\left[\theta_{-}\right]=1 /$ normalizationFactor $1 \mathrm{q} 1 /(\pi(3(a+b)-\sqrt{ }((3 a+b)(a+3 b))))$

$1 / 2(a+b)(a b) /\left((a \operatorname{Sin}[\theta])^{\wedge} 2+(b \operatorname{Cos}[\theta])^{\wedge} 2\right)^{3 / 2}$;

To evaluate the electric field of the charged ellipse along its vertical symmetry axis through the center at a height $z$ we calculate first the electrostatic potential $V(z)$ applying,

$$
V(z)=k \int \lambda(\theta) \frac{1}{\text { distance }(z)} \mathrm{d} \ell,
$$

where in (2) the distance $(z)=\sqrt{z^{2}+(a \cos [\theta])^{2}+(b \sin [\theta])^{2}}$. Substituting the latter in (2) and the rest of the terms i.e. the above noted, $\lambda(\theta)$ and $\mathrm{d} \ell$ results an integral that Mathematica is incapable evaluating symbolically; this is a CPU expensive procedure. To overcome this issue, we apply our method introduced and outlined in [1]. In short, we introduce a numeric integrated value of (2) for a set of $z$-values. We then fit the output with a modeled analytic $z$-dependent function making the calculation of the electric field feasible according to $\boldsymbol{E}=-\nabla V(z)$. The Mathematica code accomplishing this is as follows (Figure 3 ).

potentialintegrand $1\left[z_{-}, \theta_{-}\right]=\lambda 1[\theta] 1 / \sqrt{ }\left(\mathrm{z}^{\wedge} 2+(\mathrm{a} \operatorname{Cos}[\theta])^{\wedge} 2+(\mathrm{b} \operatorname{Sin}[\theta])^{\wedge} 2\right)$ $\sqrt{ }\left((\mathrm{aSin}[\theta])^{\wedge} 2+(\mathrm{b} \operatorname{Cos}[\theta])^{\wedge} 2\right) /$. values 1 ;

$\mathrm{V} 1\left[\mathrm{z}_{-}\right]=$Table $[\{\mathrm{z}, \mathrm{NIntegrate}[$ Evaluate[kpotentialintegrand1[z, $\theta] /$.values1], $\{\theta, 0,2 \pi\}]\},\{z, 0,10,1\}]$

As shown, the potentials, $V(z)$ beyond $z=4 \mathrm{~m}$ irrespective of the size of the ellipse, $\{a, b\}$ are indistinguishable. There are differences at shorter heights. The 


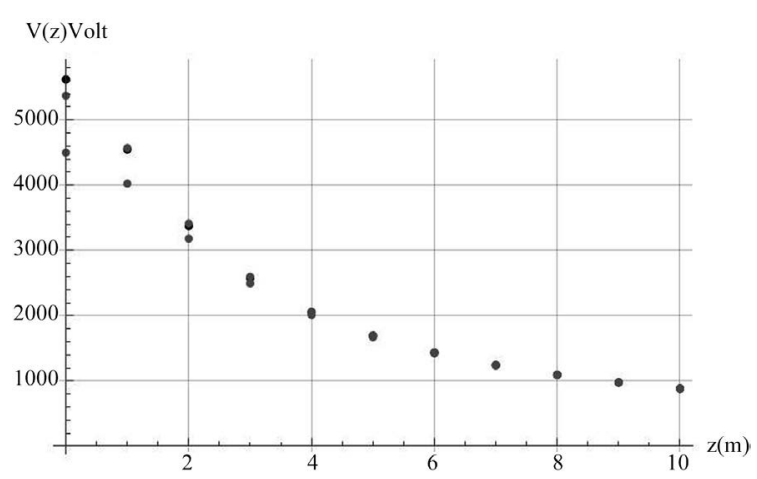

Figure 3. Dots are the numeric values of the Equation (2) for a set of $z$-values. The displayed lowest set in gray is the potential, $V(z)$ for the circular ring with $\epsilon=0$. The top set is for the ellipse with eccentricity of 0.96 . The middle set is for the ellipse with eccentricity 0.8 .

differences are not ignorable yet have common similarities. The lowest set describes the ring with the least potential. This sounds, because intuitively the ring has the largest size $a=b=2.0$ corresponding to the largest distance $(z)$. On the contrary the top data set has the strongest potential associated with the pinched ellipse with the shortest distance $(z)$. The observed similarities lead to exploring one unique analytic model function fitting the data. It appears at smaller $z$ s the potential behaves as Gaussian and at far distances fall off exponentially. With these observed featured and with some trial and error we build the model accordingly as:

$$
\begin{aligned}
& \operatorname{modelz} 1\left[\mathrm{z}_{-}\right]:=\mathrm{c} 1+\mathrm{d} 1 \mathrm{z}+\mathrm{e} 1 \mathrm{e}^{\wedge}\left(-\mathrm{f} 1 \mathrm{z}^{\wedge} 2\right) \\
& \text { fitV1z=FindFit }[\mathrm{V} 1[\mathrm{z}], \operatorname{modelz} 1[\mathrm{z}],\{\mathrm{c} 1, \mathrm{~d} 1, \mathrm{e} 1, \mathrm{f} 1\}, \mathrm{z}]
\end{aligned}
$$

We then plot the data and the fitted modeled functions.

$$
\text { plotfitdataz1 }=\text { Plot [modelz1 [z]/.fitV1z, }\{\mathrm{z}, 0,10\}, \text { PlotStyle } \rightarrow \text { Black,AxesOrigin }
$$
$\rightarrow\{0,0\}]$;

Figure 4 displays the data and the modeled fitted analytic functions. As mentioned, the fitted functions are Gaussian, $\mathrm{e}^{-z^{2}}$ at short distances with extended tails at long distances with modified linear terms.

Next, we apply the fundamental relationship, $\boldsymbol{E}=-\nabla V(z)$ to calculate the electric field assisting to calculate the force, $\boldsymbol{F}=q_{2} \boldsymbol{E}$. These are coded accordingly,

$$
\begin{aligned}
& \text { Efield1=-D[Evaluate[modelz1[z]/.fitV1z], }\{\mathrm{z}, 1\}] \text {; } \\
& \text { Eforce1=(q2/.values1)Efield1//Simplify; }
\end{aligned}
$$

Figure 5 is the graph of these forces.

As shown all three modeled functions have the same force value at the shorted height and do overlap indistinguishably at high heights. In the mediocre heights they reach a local maximum. The weakest of the three is the dashed curve and is the one associated with the circular ring as commented previously. Figure 5 shows the impact of the uneven charge distribution which is one of our objectives. The abscissa of the local maximum slides towards the center of the ellipse, nothing the larger the eccentricity the closer is the max to the center; this hasn't been reported in literature! 


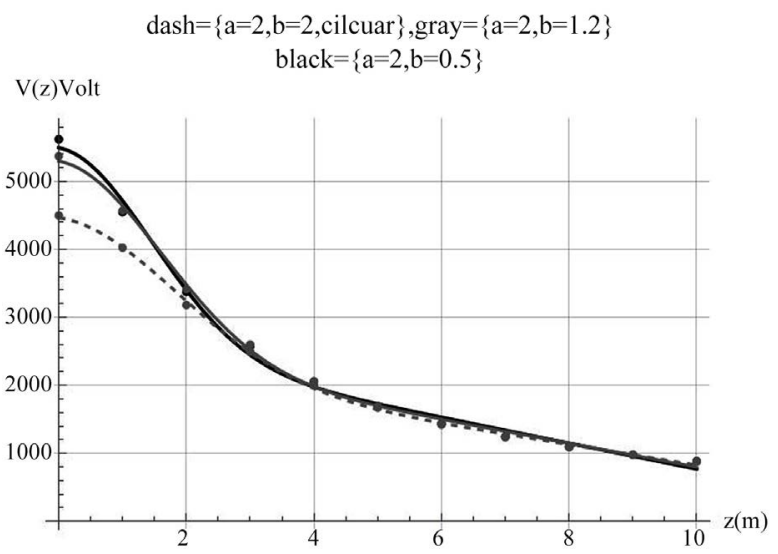

Figure 4. Three fitted analytic functions and their associated data. The plot heading is descriptive.

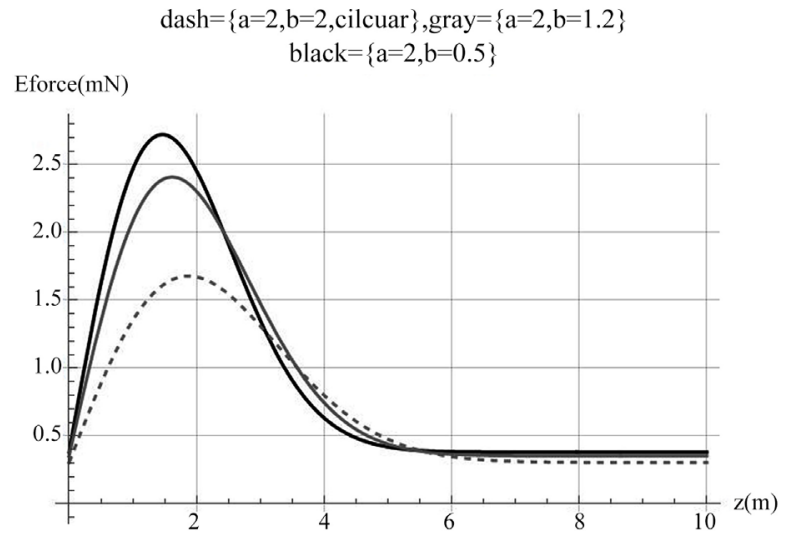

Figure 5. The curves are labeled with consistencies of Figure 4. The plot heading is descriptive.

Force axis in Figure 5 is calibrated in $\mathrm{mN}$ units. This gives a hint that what the mass range of the charged particle should be. With trial and error, we determine an appropriate mass value; its value is embedded in the listing values 1 it is in the gram. With this insight we seek for the equilibrium position, $F_{\text {electrostatic }}=m g$. Solving this equation yields the coordinate.

\section{Solve[Eforce1 $==\mathrm{mg} /$. values $1, \mathrm{z}]$}

\section{$\{\{z \rightarrow 0.23\},\{z \rightarrow 3.4\}\}$}

As shown, for the selected mass there are two associated equilibrium positions. This is meaningful because the force shown on Figure 5 has a local maximum. Consequently, any force graphed horizontally with an ordinance between the min and max force interests the force curve at two distinct points.

Now we set up the equation of motion. Applying Newton's force law,

$\boldsymbol{F}_{n e t}=m \ddot{z}(t)$ with the standard notation that the supper double dots are the acceleration. The $\boldsymbol{F}_{\text {net }}=\boldsymbol{F}_{\text {electric }}-\boldsymbol{m g}$. The needed code is,

EquationOfMotion $1=\mathrm{z} 1 "[\mathrm{t}]-($ Eforce1/.z $\rightarrow \mathrm{z} 1[\mathrm{t}])(1 / \mathrm{m} /$. values1)+g/.values1;

Numeric solutions of this equation with appropriately chosen initial conditions are coded below. The initial position of the charged particle is set higher 
than the equilibrium position and is dropped freely with initial zero speed.

$$
\text { solEquationOfMotion } 1=\mathrm{NDSolve}[\{\text { EquationOfMotion } 1==0, \mathrm{z} 1[0]==5 \text {., }
$$
$\left.\left.z 1^{\prime}[0]==0\right\}, z 1[t],\{t, 0,10\}\right]$;

The output is oscillatory as expected. Plots of oscillations are shown in Figure 6.

Figure 6 shows the initial position of three cases is set at $z=5 \mathrm{~m}$. The freely dropped particle falls and oscillates along the $\mathrm{z}$-axis. The minimum of the oscillations irrespective of $\epsilon$ is about the same; $2.3 \mathrm{~m}$ with amplitude of $1.35 \mathrm{~m}$. However, what distinguishes the cases is the period of oscillations. The circular ring with even distribution (dashed gray) has a longer period than the ellipses. This feature intuitively is understandable because the circular distribution exerts the weakest of the three forces naturally relaxing the oscillations.

Having this information in hand we craft a code putting the charged particle in motion. Since the format of the journal does not allow showing the animation, we embed its snapshot. The interested reader capable of running Mathematic code may request for a copy of the code. The animation gives a fell how a nonlinear oscillation behaves and what the impact of the nonuniform charge distribution is (Figure 7).

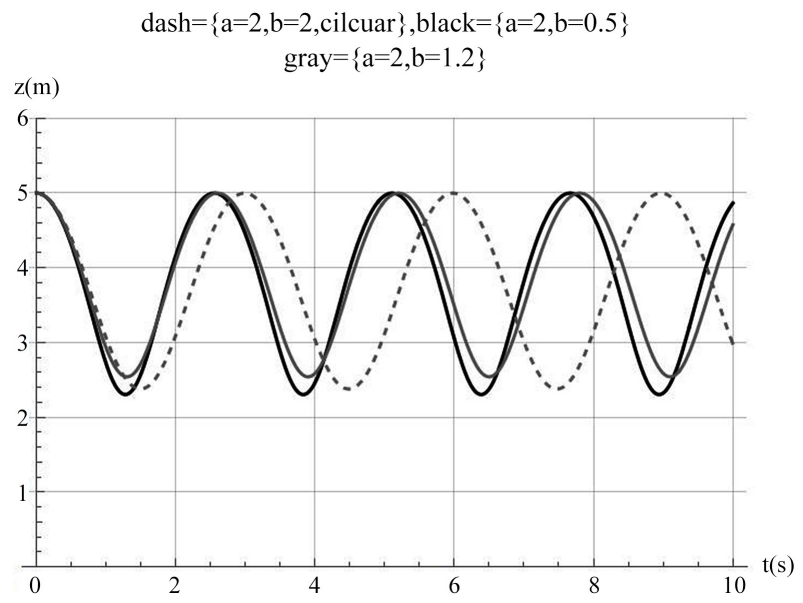

Figure 6. Oscillations of the charged particle along the z-axis vs. time. The dashed curve associated with the circular ring, the black curve with the ellipse of $\epsilon=0.96$ and the gray with ellipse of $\epsilon=0.8$.

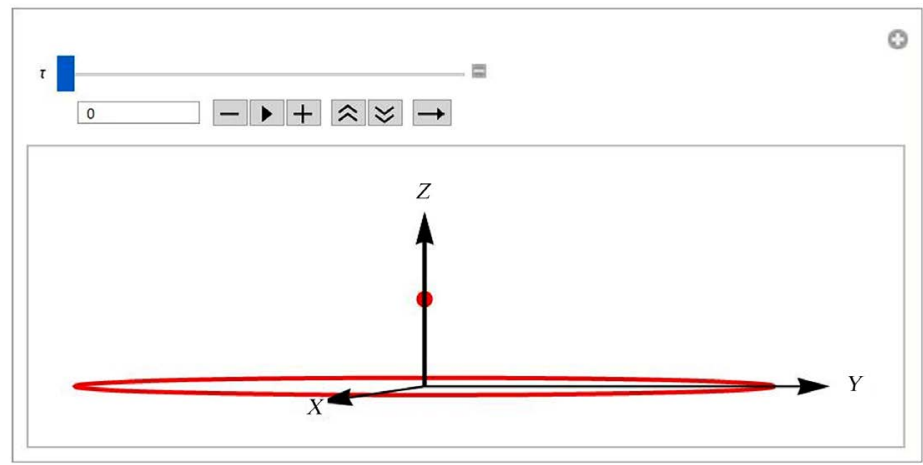

Figure 7. A snapshot of the animation of the oscillating point charge. 


\section{Discussions and Conclusions}

With a few objectives, we proposed this investigating research project. The objectives stem from the interest in the nonlinear oscillations of physical phenomena in conjunction with electrostatic related physics issues. To begin with we considered a charged ellipse. An ellipse has less geometric symmetries vs. a circle. And, naturally, the charge distribution on an ellipse inherits the same broken symmetries. In general, "the less the symmetry, the more the challenges." Distribution of charge on an ellipse is a curvature-dependent, uneven function. We have overcome the challenges caused by this unevenness conducive calculating quantities such as electrostatic potential and electric field; these are seldomly discussed in scientific literature. Having calculated these quantities, we furthered the investigation by applying them to scenarios where a loose point-like charged particle oscillates. The uneven elliptic charge distribution creates peculiar electric field and consequently makes the oscillations nonlinear. The entire calculations in this work are carried out applying Computer Algebra System (CAS for short), specifically Mathematica. In occasions, the numeric aspect of the computation surpassed the symbolic. For instance, evaluation of the electrostatic potential due to the unevenness of the charge distribution is concluded applying semi symbolic-numeric approach, similar to what we reported [1]. By the same token, the differential equation associated with equation of motion of the loose charged particle conducive to nonuniform oscillations is carried out numerically. The article embodies all the computer codes so that the interested reader familiar with Mathematic may produce our results. The codes are robust so that they may be adjusted for analyzing the "what if scenarios." For the sake of completeness, we also included a simulation of the oscillations. This is because for the sake of curiosity one wonders how an object oscillates nonlinearly! Graphs and plots of all the functions in this article are made applying Mathematica, for reproducing these graphs interested readers may find [6] [7] resourceful.

\section{Acknowledgements}

The author gracefully acknowledges the John T. and Page S. Smith Professorship funds for completing and publishing this work.

\section{Conflicts of Interest}

The author declares no conflicts of interest regarding the publication of this paper.

\section{References}

[1] Sarafian, H. (2020) Nonlinear Oscillations of a Pair of Charged Rings. America Journal of Computational Mathematics (AJCM), 10, 571-577. https://doi.org/10.4236/ajcm.2020.104032

[2] Wolfram, S. (1996) Mathematica Book. 3rd Edition, Cambridge University Press, Cambridge. 
[3] Jackson, D. (1998) Classical Electrodynamics. 3rd Edition, John Wiley, Hoboken.

[4] http://mathworld.wolfram.com

[5] Ramanujan, S. (1914) Modular Equations and Approximations to $\pi$. Quart. J. Pure Appl. Math, 350-372, 372.

[6] (2020) Mathematica V12.1.1. http://Wolfram.com

[7] Sarafian, H. (2019) Mathematica Graphics Examples. 2nd Edition, Scientific Research Publishing, USA. 